Cahiers $d u$ MONDE RUSSE

\section{Cahiers du monde russe}

Russie - Empire russe - Union soviétique et États indépendants

$46 / 3 \mid 2005$

Etrangers en Russie, Russes à l'étranger

\title{
Les français expulsés de l'empire russe par l'oukase de Catherine II du 8 février 1793
}

\author{
JULIE OLLIVIER-CHAKHNOVSKAIA
}

\section{OpenEdition}

Journals

Édition électronique

URL : https://journals.openedition.org/monderusse/8821

DOI : $10.4000 /$ monderusse. 8821

ISSN : $1777-5388$

Éditeur

Éditions de l'EHESS

\section{Édition imprimée}

Date de publication : 1 juillet 2005

Pagination : $529-543$

ISBN : 2-7132-2056-4

ISSN : $1252-6576$

Référence électronique

JULIE OLLIVIER-CHAKHNOVSKAIA, «Les français expulsés de l'empire russe par l'oukase de Catherine II du 8 février 1793 », Cahiers du monde russe [En ligne], 46/3 | 2005, mis en ligne le 01 janvier 2007, consulté le 03 septembre 2022. URL : http://journals.openedition.org/monderusse/8821 ; DOI : https://doi.org/10.4000/monderusse.8821 
chercher : repérer : avancer

Cet article est disponible en ligne à l'adresse :

http://www.cairn.info/article.php?ID REVUE=CMR\&ID NUMPUBLIE=CMR 463\&ID ARTICLE=CMR 463 0529

Les français expulsés de l'empire russe par l'oukase de Catherine II du 8 février 1793

par JULIE OLLIVIER-CHAKHNOVSKAIA

Editions de I'EHESS | Cahiers du monde russe

2005/3 - Vol 46

ISSN 1252-6576 | ISBN 2713220564 | pages 529 à 543

Pour citer cet article :

—OLLIVIER-CHAKHNOVSKAIA J., Les français expulsés de l'empire russe par l'oukase de Catherine II du 8 février 1793, Cahiers du monde russe 2005/3, Vol 46, p. 529-543.

Distribution électronique Cairn pour les Editions de l'EHESS.

(C) Editions de l'EHESS. Tous droits réservés pour tous pays.

La reproduction ou représentation de cet article, notamment par photocopie, n'est autorisée que dans les limites des conditions générales d'utilisation du site ou, le cas échéant, des conditions générales de la licence souscrite par votre établissement. Toute autre reproduction ou représentation, en tout ou partie, sous quelque forme et de quelque manière que ce soit, est interdite sauf accord préalable et écrit de l'éditeur, en dehors des cas prévus par la législation en vigueur en France. Il est précisé que son stockage dans une base de données est également interdit. 


\section{LES FRANÇAIS EXPULSÉS DE L'EMPIRE RUSSE PAR L'OUKASE DE CATHERINE II DU 8 FÉVRIER 1793*}

L'attitude de Catherine II envers la Révolution française a fait couler beaucoup d'encre ${ }^{1}$. Les paradoxes d'une souveraine, ouvertement admiratrice des Lumières et en même temps autocrate à la tête d'un immense empire peuplé de serfs, n'ont cessé de provoquer l'étonnement. Sans revenir sur cette question, il est important de rappeler que Catherine II n'éprouve aucune sympathie à l'égard de la Révolution et souhaite clairement le retour à l'ordre ancien. Cependant les relations diplomatiques entre les deux pays, même si elles se refroidissent considérablement, subsistent jusqu'au mois de juillet 1792, date à laquelle le chargé des affaires de France, Edmond Genet, est prié de quitter l'empire. L’Impératrice choisit le camp des princes émigrés, auxquels elle apporte son soutien, notamment financier. En revanche l'aide militaire souvent promise n'est pas envoyée : la France est loin et les affaires de la Pologne et de l'Empire ottoman sont plus urgentes.

L'événement qui bouleverse Catherine II est bien évidemment la mort de Louis XVI. Comme si l'Impératrice prenait soudain conscience de la gravité de la Révolution, et surtout de la menace qu'elle représentait pour son propre pouvoir.

\footnotetext{
* Cette mise au point s'appuie sur des documents inédits consultés dans différentes archives russes, dans le cadre d'une thèse sur les Français en Russie sous Catherine II et Paul I ${ }^{\mathrm{er}}$, en cotutelle entre l'université de Bordeaux 3 sous la direction de M. Figeac, et l'Académie des sciences de Russie sous la direction de M. Čudinov, avec le soutien d'une bourse Lavoisier du ministère des Affaires étrangères français. Je remercie M. Bovykin pour ses remarques constructives, et M. Rjéoutski pour ses commentaires et la mise à disposition de plusieurs notices bibliographiques sur quelques réfractaires, du Dictionnaire des Français en Russie, à paraître au Centre international d'étude du XVIII ${ }^{\text {e }}$ siècle à Ferney-Voltaire.

1. La bibliographie sur la Révolution française et la Russie est très importante. L'ouvrage ancien de Ch. de Larivière, Catherine II et la Révolution française, Paris : Le Sourdier, 1895, fait encore référence. Pour une bibliographie complète des ouvrages publiés en Russie, voir l'annuaire Velikaja francuzskaja buržuaznaja revoljucija. Ukazatel' russkoj i sovetskoj literatury. [La grande Révolution bourgeoise française. Index des ouvrages russes et soviétiques], M. : INION, 1987, p. 202-211.
} 
N'est-elle pas montée sur le trône en renversant son époux Pierre III en 1762 ? N'at-elle pas dû mater en 1773-1774 la révolte populaire conduite par Pugačëv ? Pour signifier son mécontentement face à ces débordements, mais aussi en partie par peur de la contagion révolutionnaire, Catherine II promulgue le 8 février 1793 un oukase qui met un terme aux relations entre les deux pays et prend des mesures sévères contre les Français résidant en Russie ${ }^{2}$. Elle invite ceux-ci à quitter l'empire, sauf s'ils prêtent serment de fidélité à la Monarchie, soit devant un prêtre, soit devant les autorités du gouvernement local. La première phrase est ainsi formulée :

Je soussigné jure devant Dieu Tout-Puissant et sur son Saint Evangile, que n'ayant jamais adhéré de fait ni de volonté aux principes impies et séditieux introduits et professés maintenant en France, je regarde le gouvernement qui s'y est établi comme une usurpation et une violation de toutes les lois, et la mort du Roi Très-Chrétien Louis XVI comme un acte de scélératesse abominable et de trahison infâme.

Les paroles qui suivent engagent les Français à vivre dans la religion, à reconnaître le successeur légitime de la Couronne, à se soumettre aux lois de l'empire russe, et à rompre toutes relations avec la France, tant que l'ordre n'aura pas été rétabli. En outre, les signataires se rangent officiellement du côté des ennemis de la Révolution, puisque les journaux publient leurs noms, ce qui théoriquement devrait rendre tout retour dans leur patrie impensable. Cet engagement n'est donc pas à prendre à la légère, et ceux qui s'y refusent ont trois semaines pour régler leurs affaires, à partir du moment où l'expulsion leur a été signalée. En s'appuyant sur les listes des assermentés publiées, et sans doute incomplètes, on peut estimer qu'au moins 2430 individus jurent obéissance et se placent temporairement sous la protection de l'Impératrice de Russie ${ }^{3}$. En revanche on peut se demander combien de Français

2. L'oukase du 8 février 1793 ainsi que le serment sont publiés en russe dans le journal de la capitale, les Sankt-Peterburgskie vedomosti, [cité infra $S P b V$ ], n ${ }^{\circ} 13$ du 15 février 1793. Il existe une traduction en français imprimée, voir par exemple Archives centrales historiques de Moscou [Central’nyj istoričeskij arhiv Moskvy - CIAM], f[ond]. 16/1, «Chancellerie du Général-Gouverneur de Moscou », op[is']. 457, «Sur l'expulsion de Russie des Français. 30.01-22.08.1793 ». Récemment ces textes ont été republiés en annexe de l'article de D. A. Rostislavlev, S. L. Turilova, «Francuzy v Rossii v 1793 godu» [Les Français en Russie en 1793], Cahiers du Monde russe, 39 (3), 1998, p. 304-320.

3. Les rapports sur les signataires sont réunis dans un dossier unique aux Archives de politique étrangère de l'empire Russe [Arhiv vnešnej politiki Rossijskoj Imperii - AVPRI], f. 2/1, « Collège des Affaires étrangères », op. 1293, « Affaire des Français en Russie, qui ont prêté le serment requis par l'oukase impérial du 8 février 1793 ». Les listes de ce dossier ont été imprimées en allemand dans trois fascicules. En outre elles ont aussi été publiées dans les gazettes russes. Ces matériaux ont attiré l'attention de plusieurs historiens, comme A. G. Brikner [Brückner], «Sankt-Peterburgskie vedomosti vo vremja Francuzskoj revoljucii » [«Les SanktPeterburgskie vedomosti pendant la Révolution française »], Drevnjaja i novaja Rossija, (SPb.), n 2, 1876, ou encore K. Miller, Francuzskaja emigracija i Rossija v carstvovanie Imperatricy Ekateriny Vtoroj [L'émigration française en Russie sous le règne de Catherine II], Paris, 1931 (références bibliographiques communiquées par Vladislav Rjéoutski), mais ces analyses sont succinctes et incomplètes. Dans les travaux plus récents, 1'article déjà cité de D. A. Rostislavlev et S. L. Turilova se fonde sur le dossier des AVPRI, mais il comporte quelques erreurs et 
préfèrent passer pour des sympathisants révolutionnaires et quittent l'empire plutôt que de prêter ce serment?

\section{Une poignée de réfractaires : estimation du nombre des expulsés}

Les Français réfractaires qui sont expulsés en mars 1793 sont traditionnellement considérés comme peu nombreux ${ }^{4}$. Les très anciennes études de Charles de Larivière, et à sa suite d'Emile Haumant, évoquent 43 personnes, mais elles ne mentionnent pas leurs sources d'information. Quant au récent travail de Dmitrij Rostislavlev et de Svetlana Turilova, il s'appuie sur les listes originales des Français qui prêtent serment, et considère que seulement 18 personnes s'y refusent. En effet le dossier étudié ne s'attache pas réellement aux réfractaires, et contient seulement trois rapports qui donnent les noms de deux Français de Riga, d'une Française de Tobolsk (qui semble oubliée) et de 16 Français de Kherson qui choisissent de quitter l'empire ${ }^{5}$. Ce décompte ne peut certainement pas être complet, car il paraît évident que des Français ont aussi refusé de prêter serment à Saint-Pétersbourg et à Moscou où se trouve la plus grande partie de cette communauté.

En ce qui concerne la capitale de l'empire, Saint-Pétersbourg, il est possible de croiser deux sources d'information différentes afin d'estimer le nombre de Français qui refusent de prononcer le serment. La première d'entre elles est bien entendu le journal Sankt-Peterburgskie vedomosti ${ }^{6}$, qui paraît deux fois par semaine. Ainsi, entre le 18 janvier et le 4 mars, on y trouve la première parution des annonces de départ des réfractaires. Les lois de l'empire veulent que chaque individu qui quitte le pays, qu'il soit Russe ou étranger, fasse paraître dans le journal trois annonces consécutives, qui sont classées dans la rubrique « départs ». Ces courts avertissements donnent le nom de la personne, parfois son prénom et sa profession, rarement sa nationalité, mais en revanche elles indiquent toujours son adresse, afin que les éventuels créanciers sachent où la trouver. Les Français réfractaires ne font pas

oublis, et de plus il confond le nombre des signataires avec celui des Français en Russie. Voir aussi V. S. Ržeuckij, « Istočniki po istorii francuzskih zemljačestv v Rossii v sobranii RNB. Spiski francuzov Rossijskoj imperii 1793 g. » [Les sources de l'histoire de la communauté française en Russie dans les collections de la Bibliothèque nationale de Russie. Les listes des Français de l'empire de Russie en 1793], Kollekcii. Knigi. Avtografy. Sbornik naučnyh trudov, vyp. 5, SPb. : RNB, 2003, p. 67-77 ainsi que S. N. Korotkov, «Problema social'noj transformacii francuzskoj kolonii v S.-Peterburge v konce XVIII veka : po materialam Sankt-Peterburgskih Vedomostej » [Le problème de la transformation sociale de la colonie française à Saint-Pétersbourg à la fin du XVIII ${ }^{\text {e }}$ siècle : d'après les Sankt-Peterburgskie vedomosti], Peterburgskie čtenija-97, Sankt-Peterburg v XVIII veke, SPb., 1998.

4. Cette question n'a jamais été traitée précisément, mais il est possible de trouver une estimation du nombre des expulsés dans Ch. de Larivière, op. cit., p. 132 ; E. Haumant, La culture française en Russie (1700-1900), Paris: Hachette, 1910, p. 177, et D. A. Rostislavlev, S. L. Turilova, art. cit.

5. AVPRI, f. 2/1, op. 1293.

6. Sankt-Peterburgskie vedomosti, $1793, \mathrm{n}^{\circ} 5$ du 18 janvier au n ${ }^{\circ} 18$ du 4 mars. 
exception à cette règle et la gazette donne à leur sujet les renseignements habituels, sans préciser qu'ils sont expulsés. Elle mêle leurs noms à ceux des voyageurs russes et étrangers, sans d'ailleurs indiquer leur nationalité.

La liste publiée par le journal de la capitale est à croiser avec celle des douanes de Riga et Revel. Même s'il existe plusieurs routes pour quitter la Russie vers l'Europe, les douanes les plus importantes de l'empire sont celles de Livonie. Compte tenu de la saison, le port de Cronstadt est encore pris par les glaces, et aucun voyageur ne peut donc quitter Saint-Pétersbourg ou Cronstadt par mer. Riga est bien le point de passage obligé pour les anciens résidents de Saint-Pétersbourg. En ce qui concerne l'année 1793, les rapports se présentent sous forme de lettres expédiées toutes les semaines au collège des Affaires étrangères ${ }^{7}$, qui énumèrent les allers et retours de tous les voyageurs, désignés par leur nom et prénom, parfois leur nationalité et leur profession. Alors que le 7 mars 1793, le comte d'Artois et toute sa suite, venant de Hamm, franchissent la frontière à Riga et se dirigent vers Saint-Pétersbourg où les attend Catherine II, les Français «expulsés par l'oukase de Sa Majesté Impériale », comme le porte le registre, font la route inverse les jours suivants. Les anciens résidents de Saint-Pétersbourg qui quittent la capitale, au nombre de 39 , traversent la frontière entre le 11 et le 17 mars.

Pour Moscou, où la communauté française est tout aussi nombreuse ${ }^{8}$, la gazette de la ville et le registre des douanes de Riga et de Revel permettent les mêmes déductions. En outre, deux dossiers de la chancellerie du gouverneur conservés dans les archives municipales éclairent les intentions des Français récalcitrants ${ }^{9}$. En effet, le gouverneur civil de la ville, le général-lieutenant Petr Vasil’evič Lopuhin, adresse plusieurs rapports au gouverneur militaire, le prince Aleksandr Aleksandrovič Prozorovskij, dans les jours qui suivent l'oukase du 8 février, en dressant des listes de ceux qui refusent le serment. La plus complète d'entre elles, établie au lendemain de la cérémonie, concerne neuf personnes, et indique en outre que deux d'entre elles sont déjà parties en prenant la direction de Saint-Pétersbourg.

Cependant la liste dressée par le général-lieutenant doit être prise avec précaution, car ce n'est pas un décompte définitif et ce document ne suppose pas que les ordres d'expulsion aient été suivis. Dans le registre des douanes de Riga, seulement sept personnes de la liste dressée par Lopuhin passent la frontière les 16, 29 et 31 mars. Il faut ajouter à leurs noms ceux de quatre autres Français, qui sont considérés aussi comme expulsés, même s'ils n'étaient pas compris dans les listes adressées au gouver-

7. AVPRI, f. 2/6, op. 3515, « Rapport au collège des Affaires étrangères de l'administration de Riga au sujet des passages à la frontière des ressortissants russes et étrangers. 1791-1798 ».

8. Le dossier des AVPRI, f. 2/1, op. 1293 dresse la liste des résidents français : 829 à Saint-Pétersbourg et dans ses environs, et 940 à Moscou. En réalité ces chiffres sont certainement comparables, dans la mesure où à Saint-Pétersbourg les enfants n'ont pas prêté serment, de même que tous les Français d'origine nés en Russie. À Moscou et dans toutes les autres provinces, la définition de la nationalité française a en revanche été prise dans son sens le plus large.

9. CIAM, f. 16/1, op. 457 ; f. 16/3, op. 1, « Sur les Français, qui soutiennent la Révolution Française et quittent la Russie. 22.02.1793-21.08.1794 ». 
neur. Enfin deux individus mentionnés par Lopuhin ne figurent pas sur ce registre de douane, mais dans la mesure où ils ne sont pas non plus sur les listes des assermentés, il est fort possible qu'ils aient choisi de quitter la Russie par d'autres routes que celle de la Livonie. Il apparait donc que 13 Français refusent de prêter serment à Moscou et quittent la Russie dans le courant du mois de mars 1793. Ce chiffre est nettement inférieur à celui de Saint-Pétersbourg, alors que le nombre de résidents français est comparable. Ceci s'explique certainement par le fait que la capitale est une ville de cour et un port maritime, qui attire plus de marchands, de voyageurs, de gens de passage, alors que Moscou rassemble une population étrangère plus stable.

Au total, en additionnant les chiffres de Saint-Pétersbourg, Moscou, Riga et Kherson - il est probable qu'à Tobolsk le refus de Catherine Geker ne soit qu'un différend temporaire - , il s'avère qu'au moins 70 Français [voir la liste en annexe] ne prêtent pas le serment de fidélité à la Monarchie exigé par Catherine II selon l'oukase du 8 février 1793 et sont expulsés de Russie. Ce résultat, obtenu en recoupant plusieurs documents, est certainement très proche de la réalité, mais il reste une estimation minimale. Il ne peut d'ailleurs être question de recenser le nombre exact de Français qui choisissent de quitter l'empire. En effet on peut se demander s'il n'existe pas dans les archives des dossiers jusqu'à présent encore inconnus, qui dresseraient la liste de contestataires dans d'autres régions. Combien de Français sont partis sans se signaler, dans les jours et les semaines précédentes, ayant senti l'orage venir ? Est-il possible que certains aient choisi des douanes moins scrupuleuses pour quitter l'empire plus discrètement? En réalité ce n'est pas tant la question du nombre exact des réfractaires qui suscite l'intérêt, que les raisons de leur départ.

\section{Les Français qui quittent l'empire russe par obligation}

Avant de se pencher sur l'analyse de l'ensemble des réfractaires, il faut noter que tous ces Français ne quittent pas l'empire russe par choix, mais que quelques-uns sont forcés de partir à cause des fonctions qu'ils occupent. Cette remarque ne concerne qu'une minorité d'entre eux, puisqu'elle ne s'applique qu'à deux ecclésiastiques catholiques expulsés de Moscou et aux trois chargés d'affaires des consulats et vice-consulats de France encore présents en Russie. Ces cas éclairent cependant l'atmosphère qui règne dans le pays au moment de l'édit du 8 février 1793 .

Il faut commencer par rappeler que les seuls Français qui, d'après la formulation de l'oukase, n'ont pas la possibilité de rester dans l'empire russe et de prêter serment, sont les «Consuls français, Vice-Consuls, Agents et gens attachés à eux, de NOS deux Capitales et des autres endroits où il y en a », qui sont tout simplement renvoyés. L'article 3 fait quand même une exception pour « ceux d'entre eux qui ayant été commerçants en Russie, y ont été chargés de titres et de fonctions consulaires, et qui désireront rester pour vaquer uniquement aux affaires de commerce », à condition bien entendu qu'ils prêtent le serment. C'est notamment la situation dans laquelle se trouve Raimbert, gros négociant français de Saint-Pétersbourg, qui a plusieurs fois exercé des missions consulaires, mais en considération 
de ses importantes activités économiques il est autorisé à prêter serment dans la capitale. En revanche il n'est laissé aucune alternative au consul et aux chargés d'affaires en exercice, c'est-à-dire le consul de France à Saint-Pétersbourg, Patot d'Orflans, le vice-consul à Moscou, de Bosse, ainsi que François Billiot, chargé des fonctions du vice-consulat de Cronstadt depuis 1791. Les rapports de Lopuhin sur les réfractaires ${ }^{10}$ montrent qu'à Moscou, Jean de Bosse était depuis longtemps surveillé et devenu indésirable. Cet ancien colon avait été chargé des affaires de la communauté française en avril 1788 par le vice-consul de Moscou, Pierre Martin, en son absence, fonction qui avait été confirmée par l'ambassadeur, le comte de Ségur. Dans une lettre du 30 janvier 1793 au vice-chancelier Ivan Andreevič Osterman, Lopuhin s'indigne que de Bosse, alors que les relations diplomatiques ont été rompues avec la France, continue à exercer ses fonctions, donnant des attestations pour différentes affaires, en y apposant l'ancien tampon du vice-consulat « faute d'autre », raisons pour lesquelles il demande à ce que les activités du viceconsul soient suspendues. La situation de Patot d'Orflans et de Billiot est tout aussi délicate. Leur expulsion de Russie est donc essentiellement liée à leurs fonctions officielles de représentants du gouvernement français. Restés à leur poste après la chute de la Monarchie, ils sont de fait considérés comme sympathisants du gouvernement révolutionnaire. Ils sont expulsés d'office, ce qui d'ailleurs ne semble pas les surprendre, et aucun d'entre eux ne cherche à demeurer en Russie.

La situation des deux ecclésiastiques catholiques est différente de celle des consuls, puisque dans un premier temps la possibilité leur a été laissée de prêter serment. Il est important de remarquer que l'oukase de Catherine II est particulièrement tolérant envers les religions catholique et réformées, chaque Français prêtant serment selon le culte qu'il pratique. À Moscou, deux paroisses catholiques coexistent en $1793^{11}$ : l'ancienne église des Saints-Apôtres-Pierre-et-Paul, et l'église Saint-Louis-des-Français, nouvellement instaurée en 1790. Cette dernière suscite la colère de Lopuhin, qui explique, dans un rapport du 26 février $1793^{12}$, qu'après avoir reçu l'oukase impérial, il a appelé entre autres les abbés Forceville, Levoux, Gaillard, qui « d'après renseignements se sont avérés favorables à la nouvelle constitution française et à tous les événements ignobles arrivés à Paris ». Lopuhin leur a en conséquence « conseillé de disparaître de Russie », et d'ailleurs ceux-ci ne sont ni dans les listes du serment, ni dans celle des expulsés. À propos de l'Église catholique française, il explique qu'elle « ne possède pas de maison consacrée à Dieu, et que pendant l'office dans ce courant d'air, les Français ne respectent jamais la vénération obligatoire dans une église ». De plus il remarque que les Français s'y

10. CIAM, f. 16/1, op. 457.

11. Au sujet des églises catholiques de Moscou, voir V. S. Ržeuckij, « La colonie française et l'église catholique de Moscou à la fin du XVIII' siècle », dans Cahiers du Monde russe, 41 (4), 2000, p. 615-628 et Idem, « Francuzskaja kolonija v Moskve v carstvovanie Ekateriny II » [La colonie française de Moscou sous le règne de Catherine II], in P. Čerkasov, éd., Rossija i Francija, XVIII-XX veka, vol.5, M. : Nauka, 2003. L'incident de l'expulsion des prêtres y est rapporté d'après d'autres matériaux d'archives.

12. CIAM, f. 16/3, op. 1. 
rendent finalement très peu, et plus pour discuter que pour prier. Il conclut en écrivant qu' « au contraire dans l'église catholique allemande tout est comme il faut, et quand les Français y étaient attachés, il n’y avait aucun désordre. Il serait vraiment utile de détruire l'église française, et ainsi les réunions de Français ». Cette solution radicale n'est pas mise en pratique, mais il semble que ce rapport soit repris par Prozorovskij, et même amplifié, dans une lettre qu'il adresse à l'archevêque de Mohilev, Sestrencewicz ${ }^{13}$. Finalement les statuts de l'église Saint-Louis sont modifiés, celle-ci étant dorénavant subordonnée à l'ancienne paroisse Pierre-et-Paul, et la séparation par nationalité disparaît.

Toutefois Lopuhin a besoin des prêtres catholiques pour faire prêter le serment à la communauté française de Moscou. Il écrit dans un autre rapport à Prozorovskij ${ }^{14}$ :

ont été amenés à l'administration du gouvernement, les prêtres catholiques de l'église Pierre-et-Paul, qui se trouve dans le quartier allemand, le supérieur originaire de Strasbourg Schauer, et les abbés français Pen de Matinicourt et Moisant. D'eux d'entre eux, Schauer et Matinicourt, après avoir écouté la volonté suprême de Sa Majesté Impériale, ont déclaré que se plier aux ordres ciécrits va contre leur conscience. Le troisième Moisant a affiché sa volonté de rester en Russie et a donné son serment.

Lopuhin affirme qu'André Schauer et Pesme de Matignicourt sont favorables aux idées révolutionnaires. Il est en fait difficile d'expliquer leur geste. Si le général Lopuhin y voit le signe de leur « dépravation » et de leur sympathie pour la Révolution française, il faut prendre ses explications avec prudence. Il est probable que leurs raisons sont plus religieuses que politiques : prêter un serment imposé par une souveraine étrangère et orthodoxe, puis le faire prêter à toute la communauté française, et notamment aux non-croyants, va contre leurs principes. Cet acte peut être considéré comme un blasphème dont ils ne veulent pas être complices. Le supérieur Schauer de l'église allemande regrette finalement sa décision, puisque dès le lendemain de l'acte collectif, il présente une supplique à Lopuhin, promettant de prêter serment à son tour. Le gouverneur civil doute toutefois de sa sincérité et note à son sujet que c'est un «mauvais prêtre, un prêcheur incompétent, et un homme aux mœurs peu calmes ». Sur son avis, Catherine II refuse à Schauer l'autorisation de jurer fidélité et l'expulse de Russie. Quant à Pesme de Matignicourt, premier archiprêtre de l'église Saint-Louis, que le gouverneur militaire de Moscou déteste particulièrement, il ne semble pas regretter sa décision. D'après le rapport de Prozorovskij à l'archiprêtre de Mohilev étudié par Vladislav Rjéoutski, il écrit une lettre à Catherine II en formulant ses objections contre son oukase, ce qui est une raison de plus pour l'expulser. Ces deux ecclésiastiques sont donc forcés de quitter la Russie, tandis que Moisant est chargé de la cérémonie du serment de fidélité à la Monar-

13. Lettre reproduite dans V. S. Ržeuckij, «La colonie française et l'église catholique de Moscou... », art. cit., p. 622-623. L'église, selon les termes de Prozorovskij, réunit « la canaille française », et dans la cour une sorte d'auberge a été ouverte où il est possible de lire les journaux et discuter politique autour d'une boisson.

14. CIAM, f. 16/3, op. 1. 
chie, ce qui lui vaut d'obtenir la place de desservant de l'église Saint-Louis-desFrançais ${ }^{15}$.

\section{Le libre choix de quelques réfractaires et leurs motivations}

Si les fonctionnaires consulaires français et deux ecclésiastiques de Moscou sont expulsés de Russie plus à cause de leurs fonctions que de leurs convictions politiques, qu'en est-il des autres Français qui quittent l'empire à la suite de l'oukase du 8 février? Dans son article 5, ce texte invite les signataires à ne pas prendre le serment à la légère, et insiste sur le fait que ceux qui s'y refusent « reconnaissent le gouvernement actuel de leur pays et s'y soumettent». Ces Français récalcitrants sont de fait assimilés aux Révolutionnaires, c'est-à-dire qu'ils soutiennent la politique du nouveau gouvernement en France, et qu'ils sont contre la Monarchie. La liste des réfractaires ainsi que les quelques indications qui l'accompagnent permettent d'étudier ce groupe dans sa composition. Par ailleurs, on connaît les activités et les opinions de certains d'entre eux, ce qui permet de mieux cerner leurs motivations à quitter l'empire. Ces Français éprouvent-ils réellement de la sympathie pour la Révolution et leur intention est-elle de regagner la France ?

La première remarque qui s'impose à la lecture de cette liste porte sur l'aspect purement démographique. À Moscou et à Saint-Pétersbourg, sur 52 personnes, 14 sont des femmes, dont cinq sont en couple et accompagnent leurs maris. Il faut donc constater la diversité des cas puisque, si le nombre des expulsés compte une majorité d'hommes célibataires, quelques femmes seules prennent aussi la décision de partir. Les cinq couples qui partent n'ont pas d'enfants, mais en revanche, dans la capitale, six enfants suivent leur père ou leur mère hors de l'empire.

Les journaux indiquent parfois la profession des expulsés, sans que cela soit systématique. À Saint-Pétersbourg, la gazette mentionne les activités de neuf hommes, commerçants, domestiques et artistes, ce qui montre la variété des occupations professionnelles et permet de constater que les réfractaires ne se recrutent pas dans des professions spécifiques. De plus, les journaux indiquent aussi le domicile des Français sur le départ, mais cette information est peu exploitable. Non pas qu'il soit difficile de localiser les maisons indiquées, car les explications sont très claires, mais en réalité l'intérêt n'est pas tant de situer ces logements que de définir leur niveau de confort. Or toutes les maisons nobles louent des chambres ou des appartements, plus ou moins grands et équipés, et donc le fait de résider chez quelque prince ne signifie en aucun cas être son invité. Il est de plus tout à fait possible que ces déclarations soient fausses. Plusieurs personnes sont recensées comme vivant à l'auberge, ce qui pourrait être une indication permettant d'affirmer que celles-ci ne sont que de passage. Toutefois il faut encore être prudent, puisque par exemple Bousson de

15. Place qu'il occupe jusqu'en novembre 1797. Voir CIAM, f. 16/1, op. 1391 «Plainte de l'ancien prêtre Moisant envers l'actuel syndic de l'église Saint-Louis pour non-paiement de son traitement. 9-21.12.1800». 
Mairet, qui déclare habiter à l'hôtel de «Londres », réside depuis plusieurs années à Saint-Pétersbourg, comme précepteur dans diverses familles nobles. L'indication la plus instructive fournie par ces gazettes est en réalité la date de publication des premières annonces. Tous ces Français ne se décident pas en même temps à quitter la Russie et leurs intentions sont publiées dans différents numéros du journal de la capitale. Il est important de constater que six personnes annoncent leur départ dès le mois de janvier, donc avant la promulgation de l'oukase du 8 février, ce qui indique qu'elles avaient déjà l'intention de quitter la Russie, et pourtant leurs noms sont associés à ceux des expulsés dans la liste des douanes. Les cérémonies du serment commencent le 15 février, et elles s'échelonnent sur plusieurs jours, ce qui donne aux Français le temps de réfléchir. Les moins pressés à se plier à l'oukase ne prêtent serment que le 26 du même mois, et certains Français ne pouvant se déplacer pour cause de maladie ne le feront que beaucoup plus tard ${ }^{16}$. Ainsi les réfractaires ont tout le temps de peser leur décision, sans forcément annoncer leurs intentions au lendemain de l'oukase. Quatorze d'entre eux attendent le 25 février pour faire publier leur départ dans la gazette, et quatre ne se décident qu'au mois de mars. Il est probable que certains ont hésité avant de faire le choix de partir. De même, quelques-uns ont pu prétexter une maladie imaginaire, puis finalement se résoudre à prêter serment.

Il est cependant difficile de pousser plus avant l'étude de ce groupe sans avoir recours aux cas particuliers. La liste montre que les marchands sont particulièrement nombreux parmi les réfractaires. La situation est en réalité très différente entre ceux qui ont leur maison de commerce en Russie comme Raimbert, et ceux qui pratiquent le commerce avec la Russie, mais sont établis ailleurs en Europe. Ces derniers, qui sont venus dans l'empire régler quelques affaires, n'ont aucune intention de rester en Russie et de renoncer à la possibilité de commercer avec la France. Comme ils sont simplement de passage, leur départ ne présente pas de difficulté. Ainsi il est probable que les Français Jean et Louis Reboul, qui figurent sur la liste de Moscou où la profession des expulsés n'est pas mentionnée, soient des marchands appartenant à la famille du négociant de Montpellier Reboul qui avait fondé une maison de commerce en 1783 à Kherson et qui était décédé en Crimée en 1786. Louis Reboul n'est pas installé en Russie, puisque le registre des douanes de Riga indique qu'il est arrivé de Bâle le 5 novembre 1792. D'ailleurs avant même de recevoir l'ordre d'expulsion de Moscou, les deux Reboul sont déjà partis pour Saint-Pétersbourg, et ils quittent rapidement la Russie le 16 mars, ce qui montre qu'ils avaient certainement préparé leur départ. Dans la liste de Saint-Pétersbourg, la gazette mentionne trois marchands, Joseph Bouché, Pierre Neyral et Henry Weyer, auxquels il faut ajouter Sébastien Cholé, mentionné comme marchand sur le registre des douanes de Riga lors de son arrivée dans l'empire le 26 octobre 1792.

16. Voir AVPRI, f. 2/1, op. 1293. Les rares orthodoxes prêtent par exemple serment plus tard, en mars, certainement parce que, dans un premier temps, ils ne se sont pas sentis concernés par l'oukase, car ils sont nés ou résident depuis des années en Russie. Dans le rapport sur SaintPétersbourg, arrêté à la date du 20 avril 1793, 12 personnes sont mentionnées comme malades, mais elles ont exprimé par écrit leur intention de prêter serment. Un second rapport du 14 septembre 1793, montre que neuf d'entre elles ont réellement prêté serment (les trois autres ont disparu) en même temps que 18 nouveaux arrivants Français pour cette période. 
Il n'est enfin pas exclu que d'autres réfractaires soient aussi des marchands. Il est évident que les intérêts de ces six Français sont ailleurs qu'en Russie et que leur décision de ne pas prêter serment n'a rien à voir avec leurs convictions politiques. Leur situation est exactement la même que celle des deux skippers et des sept matelots de Kherson, dont le bateau aborde en Crimée au moment de l'oukase, mais qui n'ont aucune raison de prêter serment et de rester en Russie.

En ce qui concerne les marchands français implantés en Russie de longue date, leur choix n'a pas forcément été facile. Raimbert, dont la maison de commerce fait des affaires partout en Europe, peut se permettre de rompre toute relation avec la France. Mais en revanche ceux qui font commerce de marchandises françaises s'exposent à voir leur avenir compromis en Russie. Ainsi Louis Anthoine, de la maison de négoce de son frère Antoine Anthoine, préfère quitter la Crimée, dans la mesure où son établissement pratique exclusivement le trafic entre Marseille et Kherson. «L'animosité de l'Impératrice contre les Français était poussée à un tel point, que par deux ukases, elle défendit l'introduction dans ses États de toute marchandise fabriquée en France », ce qui ne peut signifier que la ruine de la maison Anthoine en Russie ${ }^{17}$. En effet, l'oukase du 8 février 1793 consacre la rupture des relations consulaires et du commerce direct avec la France, mais plus encore l'oukase du 8 avril, deux mois plus tard, en interdisant totalement l'importation en Russie de marchandises françaises, marque la fin du commerce indirect, pratiqué essentiellement par les Anglais et les Hollandais. Il est évident que ces mesures touchent particulièrement la communauté des marchands en Russie, notamment les quelque 70 Français recensés comme commerçants à Saint-Pétersbourg, même s'ils choisissent de rester, acceptant pour un temps de modifier la provenance de leurs marchandises.

Si les marchands sont attachés à leur établissement, leur magasin, leur clientèle, leurs contrats, et ne peuvent guère régler leurs affaires et quitter la Russie, il en va différemment de tous les Français qui sont au service de la noblesse et peuvent changer de place selon leur convenance, notamment les domestiques, les précepteurs et les artistes. La gazette de Saint-Pétersbourg donne les noms de deux domestiques, le cuisinier Jacques Guillaume Philippe, et le coiffeur Charles Joseph Boucherie (ou Bouchene), qui ne semblent guère avoir laissé de traces en Russie. En revanche, le précepteur Ignace Emmanuel Bousson de Mairet est plus connu et son nom est d'ailleurs le seul parmi ces expulsés à avoir attiré l'attention de Larivière, qui évoque «Bousson de Mairet, précepteur du fils de Panine, ayant rang et titre de lieutenant-colonel, et qui a été présenté à Catherine et au grand-duc $»^{18}$. Ces professions, au service de la noblesse, leur permettent une certaine liberté et une grande mobilité. De même, les artisans et artistes, dont le talent est reconnu et qui ne sont pas en peine de trouver des commandes, ne sont pas obligatoirement liés à un pays, et peuvent exercer leur art avec autant de succès en France ou ailleurs en

17. A.-I. Anthoine, Essai historique sur le commerce et la navigation de la mer Noire, Paris : Agasse, 1820, p. 241-242.

18. Ch. de Larivière, op. cit., p. 137. 
Europe. Cela est sans doute le cas pour Jean Legros, orfèvre et diamantaire, qui est déjà installé à Saint-Pétersbourg en 1791, ainsi que pour Philippe Spol, sculpteur présent à Riga au moment de l'oukase de Catherine II. En ce qui concerne les artistes, il est possible de noter la présence du peintre Jean Baptiste Corvel (ou Carouelle) et surtout de Jean-Louis Voille, particulièrement célèbre pour ses très nombreux portraits de la noblesse russe. On remarque aussi dans cette liste le couple d'acteurs Jean-Baptiste Tureau et Marie Louise Elisabeth née Duverdier. Toutes ces personnes ne sont pas passées à la postérité, mais certaines d'entre elles ont eu un destin intéressant après leur séjour en Russie, ce qui permet de mieux comprendre pourquoi elles ont refusé de prêter serment.

Les cas de Philippe Spol et de Jean-Louis Voille sont particulièrement intéressants ${ }^{19}$. Le premier, sculpteur sur bois de grande renommée, est expulsé de Riga en février 1793, mais il revient en Russie quelques années plus tard, puisqu'il participe à la préparation du couronnement de Paul Ire, et qu'il réside à Moscou en 1797 où il semble même très bien intégré. Quant à Voille, ce peintre et portraitiste effectue en 1793 son troisième séjour en Russie, puisqu'il avait résidé dans l'empire de 1768 à 1772, de 1774 à 1777 , et de 1785 à son expulsion. Ce départ précipité n'est toutefois pas définitif, puisqu'il revient à Saint-Pétersbourg sous Paul Ire, en 1797 ou 1798. Il est d'ailleurs intéressant de noter que, dès ses débuts en Russie, il a noué des liens avec la petite cour, et qu'il se prétend « peintre du grand-duc de Russie », même si ce titre n'est confirmé par aucun document officiel. Ainsi ces deux Français, dont il est important de souligner qu'ils sont liés au grand-duc Paul, refusent le serment en 1793, mais reviennent à l'avènement de l'empereur, et ne font alors pas de difficulté pour prêter ce serment, l'oukase du 8 février étant toujours en vigueur. Leur départ en 1793 n'est donc certainement pas un geste contre la Russie, même s'il peut être interprété comme un refus de la politique de Catherine II. Ce n'est pas non plus une preuve de leur tendance politique révolutionnaire, puisqu'ils sont proches de Paul qui éprouve autant de haine, sinon plus, que sa mère à l'égard de la Révolution en France. En réalité, on peut supposer que leur voyage était programmé d'avance ou bien qu'ils ont été pris par la crainte de perdre leur clientèle française. En considérant les mesures draconiennes mises en place par Paul Irr pour contrôler les entrées des Français en Russie, il est évident que l'empereur ne leur tient pas rigueur de leur premier refus, et que ces deux artistes n'ont pas eu d'activités politiques d'agitateurs au cours de ces années. Enfin Spol aussi bien que Voille se sont certainement rendu compte que prêter serment ne les engageait pas à rester à vie en Russie, d'autant plus qu'en 1797, ce n'est plus qu'une simple formalité et leurs noms ne sont pas publiés. Ces deux cas montrent que, malgré la rupture des communications entre les deux pays, il est tout à fait possible de circuler de l'un à l'autre et d'y séjourner alternativement. Le sculpteur Spol et le peintre Voille ne renoncent pas définitivement aux nombreuses commandes et aux succès de leur carrière artistique en Russie.

19. Les informations biographiques sur Philippe Spol (recherches de Mme Irina Efremova) et Jean-Louis Voille (recherches de Mme Ludmila Rudneva) m'ont été communiquées par V. Rjéoutski. 
L'actrice Duverdier a aussi construit sa carrière en Russie où elle a connu ses plus belles heures de gloire. Arrivée dans l'Empire avec Petr Aleksandrovič Abreskov, elle a ensuite été employée chez le prince Aleksandr Nikolaevič Golicyn, puis au début de l'année 1793 elle réside à Moscou chez le comte Buturlin. Ses fonctions auprès de ces membres de la haute noblesse sont mal connues, mais il est peu probable qu'elle soit employée à titre d'actrice. Lopuhin note qu'elle « est célèbre pour avoir ruiné beaucoup de jeunes gens », mais il est difficile de savoir si ce jugement est fondé ou non. Le 8 février 1793 [nouveau style, donc le 29 janvier v.s.], le vice-consulat de Moscou enregistre le contrat de mariage de la demoiselle Duverdier avec l'acteur Jean-Baptiste Tureau, dont la réputation est moins retentissante que celle de son épouse. À l'annonce de l'oukase du 8 février, ce couple tout juste formé refuse dans un premier temps de prêter serment, mais au lendemain de la cérémonie organisée à Moscou, se ravise, tout comme le prêtre Schauer, et adresse à Lopuhin une supplique pour qu'il leur soit permis de prêter aussi sement de fidélité. Le 26 février, le général-lieutenant informe de ce cas le gouverneur militaire de Moscou, lui demandant des ordres et expliquant qu'il a jusqu'à présent refusé leur serment, « ne pouvant être sûr, que leur changement d'avis soit réel, réfléchi, que leur serment soit fait avec un cœur pur et en accord avec leurs sentiments intérieurs ». Il évoque en même temps les mœurs dissolues de Mme Duverdier, et note que Tureau n'est qu'un « acteur médiocre », ce qui ne fait pas d'eux des criminels, mais pour le moins des individus tout à fait inutiles à l'empire. La suite de cette histoire est connue par un dossier postérieur conservé dans les dossiers de l'Expédition secrète ${ }^{20}$. Le haut procureur de Moscou, Lobanov-Rostovskij, rappelle la situation de ce couple dans un rapport du 27 juin 1798, ajoutant que « le gouverneur Lopuhin fit un compte rendu à la défunte impératrice, et suivit la décision de les expulser, ce qui fut exécuté. À ce moment, la Duverdier a été volée par son mari, qui l'a abandonnée à l'étranger et y est resté ». Toutes ces recherches sont ordonnées à la suite d'une lettre du baron Grimm envoyée le 10 juin 1798 (30 mai) d'Altona au vice-chancelier, le prince Aleksandr Borisovič Kurakin ${ }^{21}$, dans laquelle il dénonce le retour en Russie d' " une certaine dame Duverdier chassée de Russie avec son mari par ordre du gouvernement ». Il indique « qu'on m'assura que cette femme était jacobine à triple carat, que son mari avait été en France un des plus exécrables buveurs de sang de la révolution, et que c'est pour leurs principes et méfaits qu'ils avaient été chassés de Russie, d'où le mari était retourné à Paris, en laissant sa femme ici », ajoutant que celle-ci est « d'un commerce facile » et mène à Hambourg une existence peu recommandable. Il explique qu'elle a cherché en vain à obtenir un passeport d'un ministre russe et une attestation des princes français, selon le règlement, pour rentrer dans l'empire russe. Cependant «elle a trouvé le moyen de retourner en Russie, j'ignore si c'est sous son nom ou sous un nom de guerre qu'elle s'est donnée ». Suite à cette

20. Archives d'État des actes anciens de Russie [Rossijskij gosudarstvennyj arhiv drevnih aktov - RGADA],f. 7/2, «Expédition secrète », op. 3135, « Sur la Française Duverdier, qui a été expulsée de Russie et qui est de nouveau revenue à Saint-Pétersbourg. 1798 » .

21. Cette lettre est reproduite dans le dossier de l'Expédition secrète, mais l'original se trouve aux AVPRI, f. 44/4, op. 290 (d'après M. Stroev). 
lettre, la Chancellerie secrète procède à une vérification auprès de la police de SaintPétersbourg et de Moscou, mais Mme Duverdier est introuvable. Ces informations, même si elles sont à prendre avec prudence et semblent sur certains points exagérées, éclairent le parcours de ce couple dans les années qui suivent leur expulsion de Russie. La jeune femme paraît avoir été entraînée par son amour. Qu'elle soit de mœurs légères n'en fait pas une agitatrice. Ayant été trompée par son mari, elle cherche à revenir en Russie où elle a connu un certain succès, mais cette entreprise s'avère plus difficile que dans le cas de Spol et de Voille. En revanche, Tureau a certainement refusé de prêter serment dans un premier temps par convictions politiques. C'est le seul Français de la liste des réfractaires pour lequel il est avéré qu'il s'est jeté dans la mêlée révolutionnaire en quittant la Russie.

\section{En conclusion}

Si 70 Français au moins refusent de prêter serment, plus de 2400 acceptent ! Dans leur écrasante majorité, les Français qui résident en Russie en 1793 ont tout intérêt à rester sous la protection de Catherine II. Rien d'ailleurs ne les empêche dans les mois qui suivent de quitter l'empire pour un autre pays, mais les registres de douanes témoignent d'une très importante baisse des départs dans les années suivantes. En effet, la communauté se compose d'individus arrivés pour la plupart dans les années 1770-1780, certains même installés depuis plusieurs générations, avec des familles dont les enfants sont nés en Russie. Sur le plan matériel et social, ils ont souvent des situations auxquelles ils n'oseraient rêver en France. Il est difficile de poser ainsi la question en termes de fidélité à leur patrie ou de reniement. Nombreux sont ceux qui ont déjà fait le choix de s'installer en Russie pour toujours, et s'ils gardent une place pour la France dans leur cœur, leurs intérêts se trouvent dans l'empire. Il est donc évident que le serment correspond moins à une question de sensibilité politique face à la Révolution, qu'à un choix matériel objectif. D'ailleurs certains Français qui décident de rester éprouvent une véritable admiration pour la Révolution, ce qu'attestent de multiples sources ${ }^{22}$. Ils sont nombreux à scandaliser par leurs propos leurs compatriotes émigrés. Quant à la police politique, elle travaille à débusquer tous les «mauvais penseurs », comme le montre l'augmentation considérable des dossiers de l'Expédition secrète concernant des Français, même si l'on peut penser que le risque de contagion révolutionnaire est exagéré.

julie@moskovia.com ...

22. Voir notamment C. F. P. Masson, Mémoires secrets sur la Russie [...], Paris : Bertrandet, 1800-1802, ou A. Fortia de Piles, Voyage de deux Français en Allemagne, Danemark, Suède, Russie et Pologne fait en 1790-1792, Paris : Desenne, 1796, t. 4, p. 89. Aux RGADA, le fonds 7 de l'Expédition secrète renferme plusieurs auditions de Français trop ouvertement républicains. Voir J. Ollivier-Chakhnovskaia, « Les mesures prises dans l'Empire de Russie envers les Français "révolutionnaires" », à paraître. 


\section{ANNEXE}

\section{Liste des Français expulsés de Russie par l'oukase du 8 février $1793^{23}$}

Dans la ville de Riga [AVPRI, 2/1, 1293. En français et en allemand]

1. Philippe Spol (sculpteur)

2. Jean Jacques Klein

Dans la ville de Kherson [AVPRI, 2/1, 1293. En russe et en allemand]

1. Jacques Bernard Kipher

2. Antoine Garnier

3. Louis Antoine (négociant)

4. Joseph François Herin

5. Honoré Bruno Herin

6. Marcus Martin

7. Joseph Salem

8. Pierre Joseph Blanc (skipper)

9. Pierre Conovetsche (matelot)

10. Louis Laurent Bansauve (matelot)

11. Joseph Versele (matelot)

12. Jean Pierre (matelot)

13. Placide Godoe (matelot)

14. Jean Badon (matelot)

15. Pierre Joseph Coste (skipper)

16. Pierre Simon (matelot)

Dans la ville de Moscou [CIAM, 16/1, 457 ; 16/3, 1 ; AVPRI, 2/6, 3515. En russe]

1. André Schauer (ecclésiastique, supérieur) [Lopuhin] [Riga 29.03]

2. Pesme de Matignicourt (ecclésiastique, abbé) [Lopuhin] [Riga 29.03]

3. Louis Wilhem Richel [Lopuhin] [Riga 29.03]

4. Joseph Jean Baptiste Tureau (acteur) [Lopuhin] [Riga 29.03]

5. (sa femme) Marie Louise Elisabeth Tureau née Duverdier (actrice) [Lopuhin] [Riga 29.03]

6. Louis Reboul (marchand) [Lopuhin] [Riga 16.03]

7. Jean Reboul (marchand) [Lopuhin] [Riga 16.03]

8. Sébastien Lenoir [Lopuhin]

9. Nicolas Garçonneau [Lopuhin]

10. Jean de Bosse (vice-consul) [Riga 31.03]

11. (sa femme) Louise Elisabeth de Bosse née Delavalière [Riga 31.03]

23. Les localisations dans les archives ont été précisées dans les notes de l'article. Pour les listes, l'orthographe est bien évidemment difficile à établir : certaines sources en russe indiquent des phonétiques différentes, et les sources en allemand donnent une orthographe germanisée. 
12. Léonard Liber [Riga 31.03]

13. Louise Ars [Riga 31.03]

Dans la ville de Saint-Pétersbourg [AVPRI, 2/6, 3515 ; $S P b V$, 1793. En russe]

1. Jean Antoine François Renguer (ou Ringuier) [ $S P b V$ 18.01] [Riga 11.03]

2. (son fils) Antoine Renguer (ou Ringuier) [ $S P b V 28.01]$ [Riga 11.03]

3. (sa fille) Rosalie Renguer (ou Ringuier) [Riga 11.03]

4. Catherine Fortesan [Riga 11.03]

5. Marie Madeleine Bidot [SPbV28.01] [Riga 11.03]

6. (son fils) Pierre Charles Bidot [Riga 11.03]

7. (sa fille) Marie Antoinette Emilie Bidot [ $S P b V 28.01$ ] [Riga 11.03]

8. Louis Patot d'Orflans (consul) [ $S P b V 25.02]$ [Riga 11.03]

9. Joseph Bouché (marchand) [ $S P b V 25.02$ ] [Riga 11.03]

10. Catherine Louise Dandignac née Thomas [ $S P b V 25.02]$ [Riga 11.03]

11. Sébastien Chalé (ou Cholé) (marchand) [ $S P b V$ 18.02] [Riga 12.03]

12. Pierre Neyral (marchand) [ $S P b V 18.02$ ] [Riga 12.03]

13. Joseph Falkenar (ou Valkenar) [ $S P b V 25.02]$ [Riga 13.03]

14. Jacques Guillaume Philippe (cuisinier) [ $S P b V 22.02$ ] [Riga 13.03]

15. Quentin Mareise [SPbV25.02] [Riga 13.03]

16. François Renaud [ $S P b V 22.02]$ [Riga 13.03]

17. (sa femme) Marie Renaud [ $S P b V 22.02]$ [Riga 13.03]

18. Jean Laurent Mathieu [SPbV25.02] [Riga 15.03]

19. Serge Masset [ $S P b V$ 16.02] [Riga 15.03]

20. Jean Baptiste Corvel (ou Carouelle) (peintre) [ $S P b V 11.02]$ [Riga 15.03]

21. Ignace Emmanuel Bousson de Mairet (précepteur) [ $S P b V 25.02]$ [Riga 15.03]

22. Henry Weyer (marchand) [ $S P b V 1.03$ ] [Riga 15.03]

23. Charles Joseph Boucherie (ou Bouchene) (coiffeur) [SPbV22.02] [Riga 15.03]

24. Pierre Verger [ $S P b V 25.02]$ [Riga 15.03]

25. François Billiot (vice-consul de Cronstadt) [ $S P b V 1.03$ ] [Riga 15.03]

26. Jean Louis Voile (peintre) [ $S P b V 4.03$ ] [Riga 15.03]

27. Georges Fink [Riga 15.03]

28. (sa femme) Janette Fink [Riga 15.03]

29. Marie Langer [ $S P b V 25.02]$ [Riga 15.03]

30. (son fils) Etienne Langer [ $S P b V 25.02]$ [Riga 15.03]

31. Madeleine Bobri [SPbV 1.03] [Riga 15.03]

32. Bernille de Saint-Fale [ $S P b V 25.02]$ [Riga 15.03]

33. (son fils) Charles de Saint-Fale [ $S P b V 25.02]$ [Riga 15.03]

34. Jean Legros (diamantaire) [ $S P b V 25.02]$ [Riga 15.03]

35. Jean Guérin [SPbV22.02] [Riga 17.03]

36. Clément Morel [SPbV22.02] [Riga 17.03]

37. Joseph Levance [Riga 17.03]

38. Joseph Brel (ou Dreille) [SPbV 22.02] [Riga 17.03]

39. (sa femme) Louise Brel (ou Dreille) (de Sardaigne) [SPbV22.02] [Riga 17.03] 